\title{
The Adaptive Aerosol Delivery System in a Telehealth Setting: Patient Acceptance, Performance and Feasibility
}

\author{
Kurt Nikander, B.A.,' John Denyer, B.Sc., ${ }^{2}$ Mary Dodd, D.Sc., FCSP, ${ }^{3}$ Tony Dyche, B.Sc., \\ Kevin Webb, M.D., ${ }^{3}$ Peter Weller, M.D., ${ }^{4}$ and David Stableforth, M.D. ${ }^{5}$
}

\begin{abstract}
Background: The telehealth service is one of the fastest growing healthcare segments. It is increasingly utilizing computer technology and telecommunication equipment to either provide continuous vital sign monitoring or facilitate patient care at home, rather than relying solely on in-person care.

Methods: We conducted a 6-week open study in nineteen patients with cystic fibrosis enrolled from three centers, to investigate patient perception of a telehealth enabled nebulizer system (Prodose Adaptive Aerosol Delivery [AAD] System), which enabled the doorstep delivery of repeat medication.

Results: The results showed that patient confidence in the device and perception of ease of use was high with no significant change between the start and end of the trial. Views on the home delivery of medication were split between 'great' and 'inconvenient.' However, if the delivery system had been more flexible and delivered all the patients' drugs, the majority of patients would have had their medication delivered in this way.

Conclusions: The trial showed that it was possible to build telehealth technology into an advanced nebulizer system, and that patient acceptance of the technology was unlikely to be a barrier to the adoption of such a telehealth system.
\end{abstract}

Key words: Telehealth, Adaptive Aerosol Delivery (AAD) System, nebulizer, cystic fibrosis, colistin, questionnaire

\section{Introduction}

$\mathbf{T}$ HE USE OF TELECOMMUNications- and Internet-based technology in healthcare has grown rapidly during the last decade due to the recognition of its potential to improve safety, quality, and efficiency of patient care. The term "telehealth" according to the American Telehealth Association should be used as a broad definition of remote healthcare services enabled by telecommunications technology. ${ }^{(1)}$ Telehealth encompasses telemedicine, telemonitoring, and telecare. Key application areas of telehealth include Electronic Medical Records, and telecommunications- and Internetbased technologies, and services. ${ }^{(2)}$

Both telecommunications- and Internet-based technologies have recently been shown to be useful tools for interactive monitoring in patients with respiratory diseases like asthma and cystic fibrosis (CF). ${ }^{(3-5)}$ In a randomized parallel group study telecommunications- and/or Internet-based specialist monitoring and treatment of asthma patients (electronic diary) was shown to result in significantly more improvement in comparison with conventional monitoring and treatment provided by specialists and general practitioners. ${ }^{(3)}$ Telepharmacy training (interactive video) of asthma patients in the use of pressurized metered dose inhalers was shown in a randomized parallel group study to be significantly more efficient than education provided via written instructions on inhaler package inserts. ${ }^{(4)}$ The feasibility of Internet-based monitoring (spirometer connected to laptop, symptoms diary on laptop) was investigated in a case study of five patients with CF. ${ }^{(5)}$ The authors concluded that further system refinement and evaluation was required to determine patient compliance with the setup.

These studies suggest that the use of telecommunicationsand Internet-based interactions between patients with asthma

\footnotetext{
${ }^{1}$ Philips Respironics, Respiratory Drug Delivery, Parsippany, New Jersey.

${ }^{2}$ Philips Respironics, Respiratory Drug Delivery (UK) Ltd, Chichester, United Kingdom.

${ }^{3}$ Wythenshawe Hospital, Manchester, United Kingdom.

${ }^{4}$ Birmingham Children's Hospital, Birmingham, United Kingdom.

${ }^{5}$ Birmingham Heartlands Hospital, Birmingham, United Kingdom.
} 
or $\mathrm{CF}$, and healthcare providers could improve the outcomes and possibly reduce time and cost of therapies. This is especially important in $\mathrm{CF}$ in which new therapies and aggressive management of the lung disease have increased both survival and quality of life. Lung destruction in CF is caused by airway obstruction due to dehydrated secretions, endobronchial infection, and inflammation with bronchiectasis and progressive airways disease as end stages. Early intervention is therefore the norm, and the therapies are aimed at removing secretions, reducing inflammation and controlling infection. These include daily medications with inhaled antibiotics, $\beta 2$-agonists and mucus-active agents, enzyme and vitamin supplements, airway clearance sessions, and exercise. ${ }^{(6,7)}$ The timely regular supply and renewal of prescriptions is an increased burden to this patient group.

The development of the Adaptive Aerosol Delivery (AAD, Philips Respironics, Respiratory Drug Delivery, Chichester, UK) technology has created a platform for telecommunications- and Internet-based interactions between the patient, the AAD System, the pharmacy, and the healthcare professionals. The AAD System was developed with the aim to deliver precise amounts of aerosol to the patient, to provide feedback on aerosol delivery to the patient, and to record information (when, how much) on drug delivery. ${ }^{(8-11)}$ These features made the AAD System well suited for an exploratory study in CF patient acceptance and feasibility of a telehealth capable aerosol delivery system.

The primary aim of this exploratory study was to evaluate in CF patients at home the acceptability and use of an AAD System in a telehealth setting. The secondary aims included evaluation of the AAD System performance, that is, to assess the perceived value of home delivery of drug, to assess data integrity of the system, and to monitor adverse events and serious adverse events.

\section{Materials and Methods}

\section{Overall study design}

This was an open 6-week exploratory study with a group of CF patients enrolled from three centers in UK: Wythenshawe Hospital, Manchester; Birmingham Children's Hospital, and Birmingham Heartlands Hospital, both in Birmingham. The study was planned with two visits to the clinic and four electronic prescription requests for additional medication at study days 5, 15, 25, and 35. During Visit 1 the patient was trained in the use of the prototype Prodose Adaptive Aerosol Delivery (AAD) System (Prodose AAD System; Philips Respironics, Respiratory Drug Delivery, Chichester, UK) and used it for delivery of nebulized colistimethate sodium (Promixin; Profile Pharma Ltd., Chichester, UK) twice daily, after which data were downloaded via a modem to a call center (Fig. 1). ${ }^{(11)}$ The patient then filled in the first questionnaire. At study days 5, 15, and 25 the Prodose AAD System was set to prompt the patient to download the data from the Prodose Disc (AAD Disc) via the modem to the call center, which would then send an electronic prescription request to a pharmacy that would dispense the prescription plus a replacement AAD Disc (Fig. 2). At study day 35 the patient would download the data from the AAD Disc via the modem to the call center, and would then receive confirmation of study completion. During Visit 2 the patient returned his/her Prodose AAD System with the AAD Discs to the clinic and completed the second questionnaire. A telephone interview was performed during the following week.

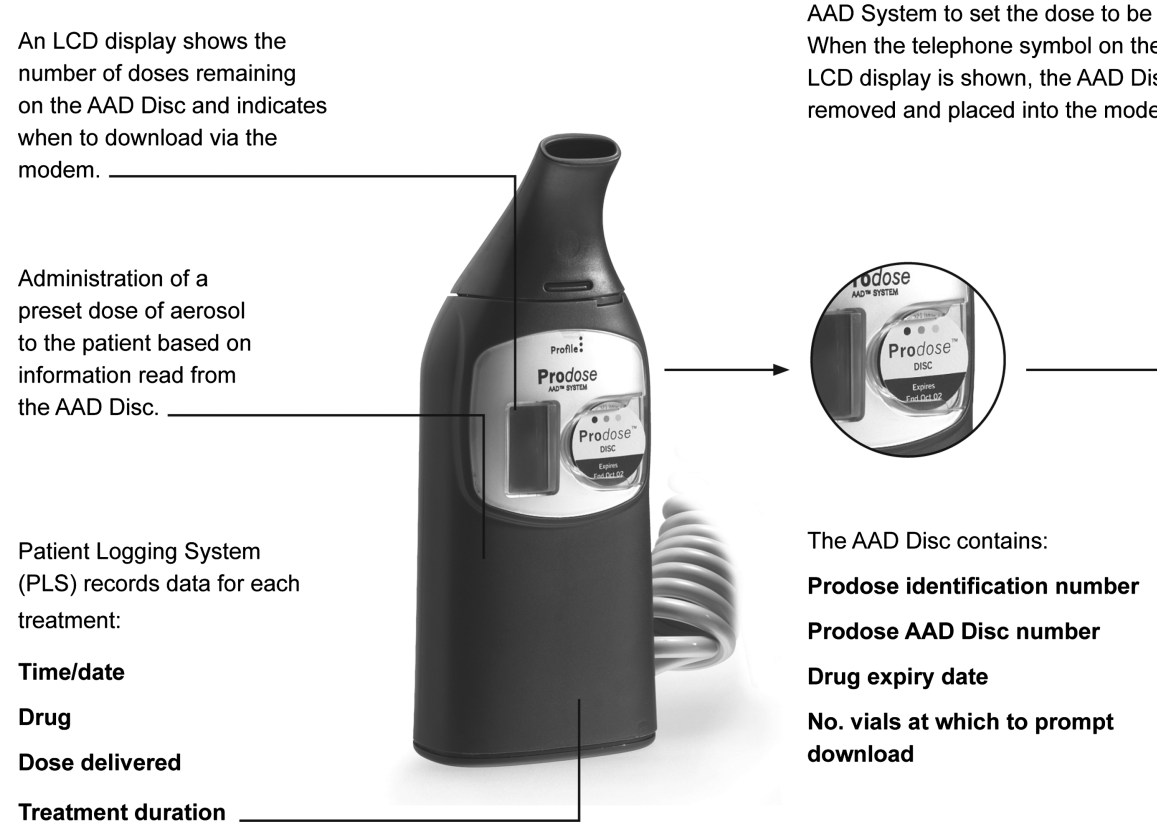

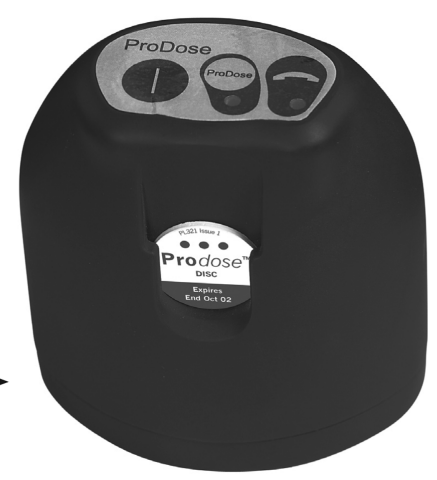

The modem connects to a standard telephone line, the AAD Disc is placed into the slot and, when the modem is activated, transmits AAD Disc data to the call center.

FIG. 1. Overview of the Prodose Adaptive Aerosol Delivery (AAD) System, the Prodose AAD Disc, and the modem used in the study. 


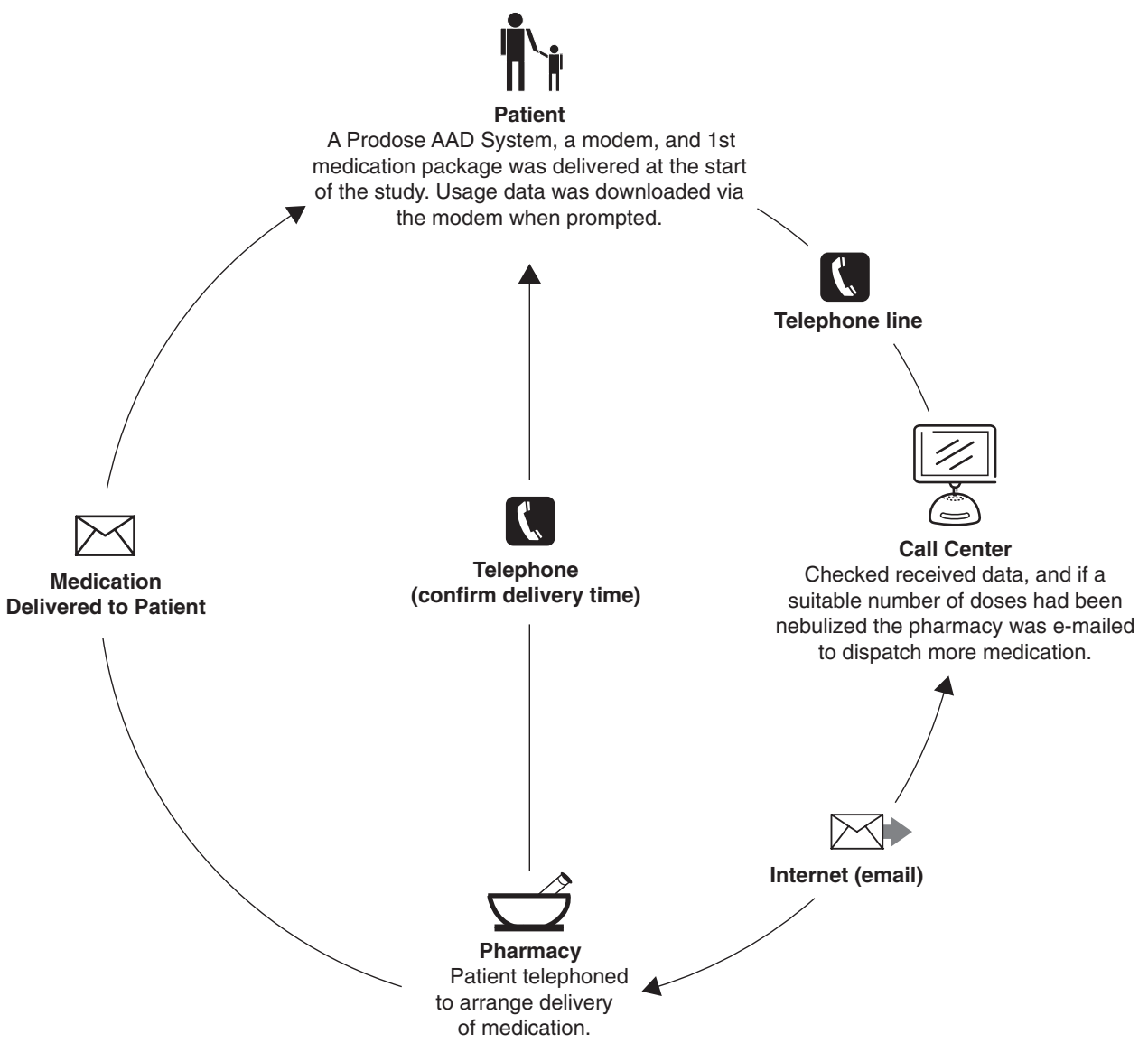

FIG. 2. Schematic presentation of the telehealth system as used in the study including all the parts in the system, that is, the patient, the call center, and the pharmacy.

\section{The Adaptive Aerosol Delivery (AAD) System}

The equipment used in the study was a prototype Prodose AAD System, which included a hand-piece, a compressor, an AAD Disc and a phone modem. When the AAD Disc was inserted into the slot on the front of the Prodose hand-piece, drug-specific data on dosage were transmitted to the handpiece by radio frequency technology. ${ }^{(11)}$ The hand-piece had an LCD display that showed battery condition, remaining treatments, when to download the AAD Disc onto the modem, and an indication of successful drug delivery. The Patient Logging System (PLS) built into the Prodose handpiece recorded time, date, drug code, dosage, and treatment time. This data could be downloaded through the infrared port on the hand-piece to a computer.

\section{The AAD Disc}

The AAD Disc was specific to the drug package and recorded both the patient's adherence to the treatment regimen and the number of nebulizer treatments. The PLS data on the AAD Disc was downloaded through the phone modem to the call center. The data consisted of the AAD Disc product identification number (PIN), the drug ID, the dosage, the drug expiry date, the download alarm value, the number of drug doses remaining on the AAD Disc, and a number used in a data checking procedure to identify errors (check number). When the correct number of vials had been used, a prescription request was sent via the modem to the pharmacy which dispensed the prescription plus a replacement AAD Disc after having contacted the patient for the most convenient time of delivery.

\section{Electronic data integrity}

The Prodose AAD System incorporated check number data validity checks on the data stored in the PLS and on the AAD Disc. On the AAD Disc the drug code and dose regimen were held in a read-only memory on the radio tag, and the validity of the data read from the tag by the Prodose AAD System was controlled by a check number specific to this block of data. The data received by the call center from the modem was also check numbered.

\section{Study questionnaire}

The study questionnaire was designed by external expertise (NOP Healthcare, London, UK) to evaluate the Prodose AAD System. The questionnaire was divided into six sections and was as follows:

1. Demographic data

2. The patients' perceptions of the Prodose AAD System at the start and end of the trial

3. The patients' perceptions of the Prodose AAD System in relation to their standard nebulizer 
4. The patients' ability to understand the symbols on the LCD display

5. The extent of use and usefulness of the information provided by the Prodose AAD System

6. Improvements to the Prodose AAD System suggested by the patients.

The questionnaire used a scale of $0-7$ for patients' confidence and ease of use with the Prodose AAD System, where 7 indicated a high confidence/ease of use. A scale of 0-10 was used to determine how patients compared the Prodose AAD System with their ordinary nebulizer.

\section{Telephone interview at the end of study}

The telephone interview was performed as a structured interview with 19 questions (Table 1) designed to evaluate the acceptability of the telehealth setup. Due to the difficulty in presenting responses to open questions, the decision was made to analyze the transcripts by reading the responses, identifying the main points raised and grouping these responses. Some patients raised several points on a question with the consequence that the number of responses on a specific question could exceed the number of patients.

\section{Patients}

The study group consisted of children over 7 years of age and adults with a confirmed diagnosis of CF. All patients had been treated with nebulized colistimethate sodium (Colomycin, Forest Ltd, Bexley, UK) twice daily for at least 3 months before inclusion, and had not had an exacerbation during the last 2 weeks before inclusion. A telecom socket in the home was a requisite to facilitate AAD Disc/modem data transfers. The study was performed in accordance with the principles stated in the Declaration of Helsinki, and was approved by the local ethics committees. The patients were invited to participate in the study by the investigators, and both verbal and written information was given to the patients.

\section{Study endpoints and statistics}

The primary endpoints were the patients' acceptability and use of the Prodose AAD System in a telehealth setting to patients at home. The evaluation of the acceptability and use were based on the questionnaires and the telephone interview. The secondary endpoints were the perceived value patients placed on the delivery service for the study drug, the data integrity of the technology, and monitoring of adverse events and serious adverse events. The patients' use of the Prodose AAD System was analyzed as part of the assessment of the data integrity of the system. Treatments were classified as invalid if the data was beyond the patient's study start and end dates, if data consisted of predespatch calibration tests or posttrial checks, if data was recorded on the original device after a replacement device had been requested, or if the patient was in hospital. As the Prodose AAD System made it possible to take pauses during treatment and still deliver the preprogrammed dose, there was no strict time schedule for the twice daily regimen.

The study numbers were rationalized on a calculation of precision, as follows. It was assumed that the overall acceptability of the device would be $\sim 80 \%$ and a $95 \%$ probability of assessing this level of acceptability would be within $\pm 15 \%$. Forty patients should have been enrolled to provide for an expected loss-to-follow-up of $25 \%$, leaving 27 patients for analyses. Demographic information has been presented as descriptive data only. Paired observations have been analyzed by calculating mean differences; $95 \%$ confidence intervals were calculated around each mean difference. An arbitrary level of 5\% significance was assumed throughout.

TAble 1. End of Study Questionnaire

Thinking now about using the Prodose System, how did you feel about using it?

What do you think were the good things, if any, about it?

What do you think were the bad things about it?

Which parts of it, if any, did you find easy?

Which parts of it did you find difficult/complicated?

To what extent did you find using the device got easier over the trial?

How many drugs/medications do you currently take on a daily basis for your cystic fibrosis?

How do you manage taking these different drugs using the same nebulizer?

How would you feel about using the Prodose for only one drug, and having to use another nebulizer for your other drug?

How would you feel about having to use a different disc for each drug, and change this as well as the drug in the chamber?

You will have noticed that there was drug left in the chamber at the end of treatment, what did you think about this?

How did the home delivery system compare to your usual system?

Before the phone call to arrange a delivery time, how confident were you that the order was made?

If you had the choice would you have all your drugs delivered like this?

What pack size do you think would be ideal for home delivery?

What pack size do you think would be ideal for collection from the pharmacy?

How much did you use the handbook that was provided with the device?

How much did you use the patient guide that was provided with the device?

How much did you use the video that was provided with the device?

Thinking about the system in general, what other concerns did you have about using it?

Is there anything else you would like to say about the Prodose AAD System? 


\section{Results}

\section{Patients}

Twenty-one patients were randomized into the study, one never started, and one withdrew 46 days after enrolment. The lower than planned enrolment was due to a late start of the study, which diminished the available time for patient enrolment. Out of the 19 enrolled patients, 6 were children ( 3 boys, mean age 11 years) and 13 adults ( 5 males, mean age 29 years). Prior to enrolment, 11 patients used a conventional jet nebulizer, 7 used the HaloLite AAD System, ${ }^{(11)}$ and 1 used an ultrasonic nebulizer. Two of the 19 patients used an alternative therapy for a short time but resumed use of colistimethate sodium via the Prodose AAD System after release from hospital. The posttrial telephone interview was not possible for 1 of the 19 patients, due to a telephone line fault. No serious adverse events occurred during the study, but three adverse events were recorded. One patient experienced chest tightness following use of the study drug, and two patients were hospitalized for a short period and then resumed the colistimethate sodium regimen. The hospitalizations were part of these patients' individualized treatments, and therefore not considered as serious adverse events. The respective study investigators did not find any relationships between the adverse events and the Prodose AAD System.

\section{Questionnaires}

The results of the questionnaire related to patient confidence with, and ease of use of, the Prodose AAD System and the AAD Disc are shown in Table 2. There were no differences between start and end of study in confidence and ease of use except for the difference in "Ease of use first time used a modem unit and Disc" $(p=0.04)$. Overall the scores were high, ranging from 6 to 7 , with the large majority of the patients not scoring any differences between start and end of study.

The results of the questionnaire related to the comparison of the patients' ordinary nebulizer with the Prodose AAD System and AAD Disc are shown in Table 3. The patients felt that the Prodose AAD System delivered more drug into the lungs $(p=0.008)$ and overall was more desirable $(p=0.03)$. The question related to the telehealth setup, that is, ordering of more drugs from the pharmacy through the AAD Disc and telephone modem showed a borderline significance $(p=0.06)$ indicating that patients' acceptance increased over time.

\section{Telephone interview at the end of study}

In general, the acceptability of the telehealth setup-the Prodose AAD System, the AAD Disc/modem, and the home delivery of the prescription drug-was positive. The portability and ease of cleaning were thought to be good, and the Prodose AAD System was seen to be easy to use. Areas that drew negative comments were the hand-piece, most probably due to instability and weight of the prototype device, and the inflexibility of the prescription drug delivery service. Thirty-nine percent of the patients thought that using a different AAD Disc for each drug was okay, whereas 50\% disliked the idea. The questions related to the telehealth service indicated that patients were split evenly on their views on whether the home delivery system was "great" $(44 \%)$ or "inconvenient" (44\%). However, in the event that the delivery system was more flexible, and delivered all the patients drugs, a majority (66\%) were in favor of having all their drugs delivered in this way. The ideal drug pack size for home delivery was thought to be 60 vials, which in most cases would equate 1 month's supply. Residual drug left in the chamber at the end of treatment was not a cause for concern, with the majority of patients saying they "just accept it."

\section{Data validity}

Data on the use of the Prodose AAD System was available from all 19 patients, with a mean of 77.5 days on treatment (median 79, range: 46-112 days). There were 1473 planned treatments with the Prodose AAD System, and the PLS recorded 1305 treatments of which 125 treatments were classified as incomplete leaving 1180 complete treatments (Table 4).

Analysis of downloads of data from the PLS in the handpiece indicated that there were no PLS data errors recorded. During the study 73 AAD Discs were used of which 71 were returned. The information on drug and dose regimen was read $100 \%$ correctly from the AAD Disc to the hand-piece.

\section{Discussion}

In this study, the primary objective was to evaluate acceptability and use of a new nebulizer system with a number of telehealth capabilities - the prototype Prodose AAD System with the AAD Disc/modem-in patients with CF. The

Table 2. Patients' Perceptions of the Prodose Adaptive Aerosol Delivery (AAD) System at the Start (BASELINE) AND THE END OF THE STUdY

\begin{tabular}{|c|c|c|c|}
\hline Questions & Baseline & End of trial & Mean difference and $95 \% \mathrm{CI}$ \\
\hline Confident using hand-piece, compressor and Disc at home & 6.47 & 6.78 & $0.32(-0.11 ; 0.74)$ \\
\hline Confident in ability to use modem unit and Disc at home & 6.52 & 6.73 & $0.21(-0.23 ; 0.92)$ \\
\hline Ease of use first time used hand-piece, compressor and Disc & 6.57 & 6.63 & $0.05(-0.58 ; 0.69)$ \\
\hline Ease of use first time used a modem unit and Disc & 6.67 & 6.88 & $0.21(0.01 ; 0.44)$ \\
\hline Ease of use of Prodose to operate hand-piece & 6.63 & 6.63 & $0.00(-0.45 ; 0.45)$ \\
\hline Ease of use of Prodose to operate modem unit & 6.73 & 6.73 & $0.00(-0.28 ; 0.28)$ \\
\hline Confident Prodose delivered drug into lungs & 6.57 & 6.31 & $-0.26(-0.88 ; 0.36)$ \\
\hline Confident using Prodose correctly & 6.63 & 6.42 & $-0.21(-0.78 ; 0.36)$ \\
\hline
\end{tabular}

CI, confidence interval 
Table 3. Results of the Comparisons between the Patients' Prestudy Nebulizers (Baseline) and the Prodose Adaptive Aerosol Delivery (AAD) System (End of Trial)

\begin{tabular}{lccc}
\hline Questions & Baseline & End of trial & Mean difference and 95\% CI \\
\hline Ease of operating & 9.26 & 8.78 & $-0.47(-1.16 ; 0.22)$ \\
Fits into daily routine & 8.78 & 8.26 & $-0.52(-1.53 ; 0.48)$ \\
Method of ordering repeat prescriptions & 7.72 & 8.72 & $1.00(-0.07 ; 2.07)$ \\
Amount of drug delivered into lungs & 6.78 & 8.68 & $1.90(0.56 ; 3.22)$ \\
Overall desirability & 7.21 & 8.73 & $1.52(0.19 ; 2.08)$ \\
\hline
\end{tabular}

CI, confidence interval.

results showed that the patients found the Prodose AAD System very acceptable and easy to use, but that the home delivery system would need to be more flexible to be acceptable by the majority of the patients. The logged data showed that information on drug and dose regimen was read $100 \%$ correctly from the AAD Disc to the Prodose handpiece. No serious adverse events occurred, but three adverse events were recorded. The study investigators, however, did not find any relationships between the adverse events and the Prodose AAD System.

With the exception of ease of use of the modem and the AAD Disc, the patient perception of the ease and confidence of use of the telehealth system did not change significantly over the course of the trial. This was probably partly a consequence of the training and instructions of patients at Visit 1, but probably also a consequence of the user friendly design of the telehealth system. The positive feedback on the setup used in the study was confirmed both in the comparison between the patient's usual conventional nebulizer and the Prodose AAD System, and in the level of patient compliance with the correct use of the Prodose AAD System. The

Table 4. Table with Data on Treatments (Planned, Missed, INCOMPlete, AND COMPlete) DOWNLOADED FROM the Built-in Patient Logging System in the Prodose Adaptive Aerosol Delivery (AAD) System

Planned Missed Treatments Incomplete Complete Patient treatments treatments in total treatments treatments

\begin{tabular}{rrrrrr}
\hline 1 & 76 & 1 & 75 & 0 & 75 \\
2 & 80 & 4 & 76 & 26 & 50 \\
3 & 78 & 27 & 51 & 0 & 51 \\
4 & 112 & 29 & 83 & 24 & 59 \\
5 & 70 & 8 & 62 & 4 & 58 \\
6 & 71 & 11 & 60 & 6 & 54 \\
7 & 80 & 0 & 80 & 1 & 79 \\
8 & 81 & 8 & 73 & 0 & 73 \\
9 & 81 & 10 & 71 & 3 & 68 \\
10 & 61 & 1 & 60 & 1 & 59 \\
11 & 81 & 1 & 80 & 0 & 80 \\
12 & 79 & 5 & 74 & 44 & 30 \\
13 & 46 & 7 & 39 & 0 & 39 \\
14 & 63 & 3 & 60 & 0 & 60 \\
15 & 75 & 3 & 72 & 3 & 69 \\
16 & 79 & 10 & 69 & 1 & 68 \\
17 & 79 & 0 & 79 & 0 & 79 \\
18 & 85 & 5 & 80 & 5 & 75 \\
19 & 96 & 35 & 61 & 7 & 54 \\
Total & 1473 & 168 & 1305 & 125 & 1180 \\
\hline
\end{tabular}

comparison showed statistically significant differences in favor of the Prodose AAD System in the patients' perceptions of the amount of drug delivered to the lungs, and the overall desirability of the system. The compliance with the correct use of the Prodose AAD System was exceptionally high (90.4\%; complete treatments divided by treatments in total $\times 100$ ) and indicated that the patients had minimal problems in the use of the Prodose AAD System.

In the questionnaire, the method of ordering repeat prescriptions obtained only borderline significance $(p=0.06)$ over pharmacy collection. The importance of the lack of flexibility of the delivery times was further emphasized in the telephone interview at the end of the study. The responses on questions related to the telehealth service showed that patients were split evenly on their views on whether the home delivery system was "great" or "inconvenient." The fact that only colistimethate sodium was delivered through the telehealth system was clearly a negative factor. However, with additional flexibility in deliveries, and all drugs delivered through a telehealth setup, the majority were in favor of a telehealth setup. Thus the study design seems to partly have been the reason for some of the resistance to the telehealth setup.

The potential of telehealth enabled nebulizers extends far beyond an increase in convenience for the patient, as it also includes a near real-time feedback to healthcare professionals and patients regarding adherence, compliance, health, and well-being indicators. Other potential advantages include savings to the healthcare system in terms of fewer hospital visits, less wasted medication, and a more flexible drug dosing in response to changes in the patient's condition. The benefits are, however, limited to nebulized drugs, as oral drugs would require a different set of telehealth technologies.

\section{Conclusions}

Our study showed that it was possible to include telehealth technologies into an AAD System to deliver telehealth benefits successfully. ${ }^{(11,12)}$ The high confidence in and perception of ease of use of the Prodose AAD System tested in this study indicated a high acceptability of the new technology showing that the technology need not be considered a barrier to the adoption of the included telehealth system. The flexibility of the medication delivery time at the patient's doorstep was, however, highlighted as being crucial to the value the patients placed upon the home delivery service. Patients also felt that to benefit from not having to go to the pharmacy, all their medication would need to be delivered in this way. This would be an entirely feasible prospect using electronic prescriptions. 


\section{Acknowledgments}

This study was conducted by the Wythenshawe Hospital (Manchester), Birmingham Children's Hospital, and Birmingham Heartlands Hospital (both in Birmingham) in cooperation with Profile Therapeutics (UK) Ltd, now Philips Respironics, Respiratory Drug Delivery (UK) Ltd. The authors acknowledge N. Smith (PS Writing Ltd, Portsmouth, UK) for his assistance with data analysis and graphics. Profile Therapeutics (UK) Ltd manufactured the inhalation devices used in the study and also sponsored the study.

\section{Author Disclosure Statement}

Kurt Nikander is an employee of Philips Respironics, Respiratory Drug Delivery, Parsippany, NJ, and John Denyer is an employee of Philips Respironics, Respiratory Drug Delivery (UK) Ltd. Mary Dodd was a consultant to Profile Pharma Ltd, a subsidiary of Profile Therapeutics (UK) Ltd, for CF-related issues during the study.

\section{References}

1. Wuorenma JK: Tele-What?: it's time to re-think the industry's terms. Telehealth World. 2008;3:7.

2. Pagliari C, Sloan D, Gregor P, Sullivan F, Detmer D, Kahan JP, Oortwijn W, and MacGillivray S: What is eHealth (4): a scoping exercise to map the field. J Med Internet Res. 2005;7:1-19.

3. Rasmussen LM, Phanareth $\mathrm{K}$, Nolte $\mathrm{H}$, and Backer V: Internet-based monitoring of asthma: a long-term, randomized clinical study of 300 asthmatic subjects. J Allergy Clin Immunol. 2005;115:1137-1142.

4. Bynum A, Hopkins D, Thomas A, Copeland N, and Irwin C: The effect of telepharmacy counseling on metered-dose inhaler technique among adolescents with asthma in rural Arkansas. Telemed J E-Health. 2001;3:207-217.

5. Magrabi F, Lovell NH, Henry RL, and Celler BG: Designing home telecare: a case study in monitoring cystic fibrosis. Telemed J E-Health. 2005;6:707-719.
6. Doring G, and Hojby N: Early intervention and prevention of lung disease in cystic fibrosis: a European consensus. J Cyst Fibros. 2004;3:67-91.

7. Flume PA, O'Sullivan BP, Robinson KA, Goss CH, Mogayzel PJ Jr, Willey-Courand DB, Bujan J, Finder J, Lester M, Quittell L, Rosenblatt R, Vender RL, Hazle L, Sabadosa K, and Marshall B: Cystic fibrosis pulmonary guidelines. Chronic medications for maintenance of lung health. Am J Respir Crit Care Med. 2007;176:957-969.

8. Nikander K. Adaptive aerosol delivery: the principles. Eur Respir Rev. 1997;7:385-387.

9. Denyer J: Adaptive aerosol delivery in practice. Eur Respir Rev. 1997;7:388-389.

10. Nikander K, Arheden L, Denyer J, and Cobos N: Parents' adherence with nebulizer treatment of their children when using an Adaptive Aerosol Delivery (AAD) system. J Aerosol Med. 2003;16:273-281.

11. Denyer J, Nikander K, and Smith NJ: Adaptive Aerosol Delivery (AAD) technology. Expert Opin Drug Deliv. 2004;1:165-176.

12. Van Dyke R, and Nikander K: Delivery of iloprost inhalation solution with HaloLite, Prodose, and I-neb Adaptive Aerosol Delivery (AAD) systems - an in vitro study. Respir Care. 2007;52:184-190.

Received on June 3, 2009 in final form, October 27, 2009

Reviewed by: David Geller

Address correspondence to: Kurt Nikander, B.A. Philips Respironics Respiratory Drug Delivery 5 Wood Hollow Road Parsippany, NJ 07054

E-mail: kurt.nikander@philips.com 
